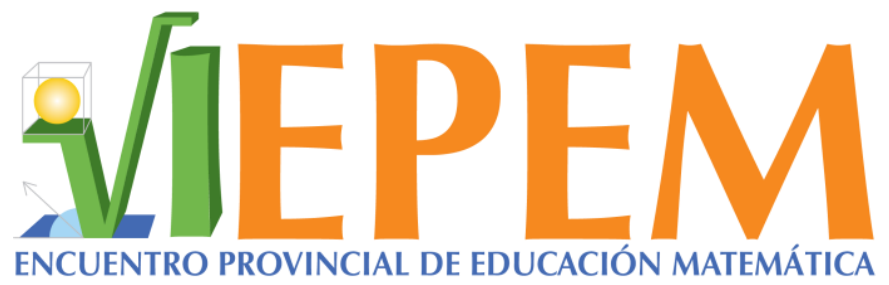

VI Encuentro Provincial de Educación Matemática.

27 al 29 de setiembre, 2017. Puntarenas, Costa Rica.

\title{
Probabilidades: Jugando y Resolviendo Problemas a través de un Viaje en el Tiempo
}

\author{
Helen Guillén Oviedo \\ hellen.guillen.oviedo@una.cr \\ Universidad Nacional \\ Costa Rica \\ Claudia Martínez Pacheco \\ claudia.martinez.pacheco@una.cr \\ Universidad Nacional \\ Costa Rica
}

\begin{abstract}
Resumen
Se pretende destacar la importancia de la Teoría de las Probabilidades como herramienta en muchas áreas del conocimiento. Se trabajará, mediante juegos y problemas, en el cálculo e interpretación de probabilidades, utilizando las propiedades básicas de esta teoría y su relación con la Teoría de Conjuntos. La historia será un eje en el taller, para lograr una correcta comprensión de la evolución de esta Teoría. Los y las docentes participantes podrán evacuar dudas con respecto a un tema que, en los últimos años, ha sido incorporado fuertemente en el currículo para la enseñanza de las matemáticas en Costa Rica. Los nuevos planes, iniciados en 2013, mencionan Probabilidad y Estadística como un área de interés e incorporan el estudio de las probabilidades desde la primaria hasta el final de la secundaria, por lo que este taller se considera importante para la formación de docentes de Segunda Enseñanza en nuestro país.
\end{abstract}

Palabras clave: Probabilidades; Ley de los Grandes Números; Bayes; Probabilidad total; Historia de las Probabilidades.

Taller

Guillén, H. y Martínez, C. (2017). Probabilidades: Jugando y Resolviendo Problemas a través de un Viaje en el Tiempo. En Y. Morales-López, M. Picado, R. Gamboa, C. Martínez, M. Castillo y R. Hidalgo (Eds.), Memorias del VI Encuentro Provincial de Educación Matemática, Costa Rica, 2017 (pp. 28-30). Heredia: Universidad Nacional. ISBN: 978-9968-9661-5-3. DOI:

http://dx.doi.org/10.15359/epem.6.6 


\section{Introducción}

El inicio de la teoría de las probabilidades se ubica alrededor de 1645 y fue bastante mundano, ya que se debió a una consulta realizada por Antoine Gombaud a su amigo Blaise Pascal sobre cómo ganar en las apuestas de un antiguo juego de azar muy popular en su época. Este juego, en apariencia muy simple, consistía en tirar dos dados 24 veces, y apostar o no a que saldría al menos un doble seis (Basulto y Camúñez, 2007). La historia cuenta que cuando Pascal se interesó por las reglas de este juego, inició por correspondencia un intenso debate con su compatriota Pierre de Fermat, por lo que intercambiaron gran cantidad de cartas en las que se hilaron por primera vez los principios básicos de las probabilidades. Posteriormente, en 1714, estas cartas serían recopiladas y publicadas por Christiaan Huygens en su libro titulado "Libellus de ratiociniis in ludo aleae or The value of all chances in games of fortune; cards, dice, wagers, lotteries, \&c., mathematically demonstrated".

El fascinante inicio de esta teoría junto con muchos otros hechos históricos interesantes alrededor de la misma, motivó la preparación de este taller, que pretende llevar a los y las participantes a través de un viaje por la historia, jugando y resolviendo problemas de probabilidades, con el objetivo de entender más fácilmente las propiedades básicas de esta teoría y su relación con la Teoría de Conjuntos.

Además, se espera que los y las participantes evacúen las dudas que puedan tener acerca de un tema muy importante en los nuevos Programas de Estudio de Matemáticas, implementados en Costa Rica desde el 2013. Se persigue también, brindar una metodología de enseñanza que los y las docentes puedan replicar en sus aulas de secundaria.

\section{Objetivo del taller}

Brindar a los y las docentes las herramientas necesarias tanto didácticas como teóricas para la enseñanza de la teoría de la probabilidad en secundaria, a través de un viaje en el tiempo por medio del uso de juegos y resolución de problemas.

\section{Metodología del taller}

En la primera etapa se pretende realizar un recorrido histórico de la evolución del cálculo de las probabilidades, utilizando juegos y problemas clásicos. Posteriormente, se presentarán algunos problemas, que permitan guiar a los y las docentes participantes en el taller a la construcción de los principales conceptos teóricos, hasta llegar a la definición clásica de probabilidad. Durante la resolución de los problemas planteados, se prestará atención a las estrategias utilizadas y a posibles errores que puedan surgir.

En la segunda etapa se busca reforzar y ampliar los aprendizajes realizados. Para reforzar la definición clásica de probabilidad se propone un problema para que sea resuelto en grupos, con el objetivo de generar una discusión que conduzca a la clausura del tema. 


\section{Resultados esperados}

Se pretende que los y las docentes conozcan algunos datos históricos acerca del desarrollo de la Teoría de las Probabilidades, de manera que puedan comprender las diferentes etapas que se dieron antes de llegar a la Teoría que actualmente conocemos.

En este taller se persigue también, que los y las docentes participantes refresquen sus conocimientos acerca de las propiedades básicas de la Teoría de las Probabilidades y su relación con la Teoría de Conjuntos, y que al terminar cuenten con una metodología de enseñanza para este tema que puedan reproducir en sus aulas.

Finalmente, se espera que los y las docentes participantes puedan evacuar cualquier duda con respecto a un tema que actualmente es de suma importancia para la enseñanza de las matemáticas en nuestro país.

\section{Referencias}

Basulto, J., \& Camúñez, J. (2007). El problema de los dados del caballero de Méré: soluciones publicadas en el siglo XVII. Suma, 56, 43-54. doi: https://revistasuma.es/IMG/pdf/56/043-054.pdf

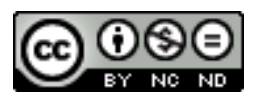

Esta obra está bajo una licencia de Creative Commons Reconocimiento-NoComercialSinObraDerivada 4.0 Internacional. 